\title{
Logistic versus linear regression-based Reliable Change Index: implications for clinical studies with diverse sample sizes
}

Rafael De Andrade Moral ${ }^{1}$, Unai Díaz-Orueta ${ }^{2}$, Javier Oltra-Cucarella ${ }^{3}$, for the Alzheimer's Disease Neuroimaging Initiative*

${ }^{1}$ Department of Mathematics and Statistics, Maynooth University, Ireland

${ }^{2}$ Department of Psychology, Maynooth University, Ireland

${ }^{3}$ Dpto. Psicología de la Salud, Universidad Miguel Hernández de Elche, Avda de la Universidad s/n Edificio Altamira, 03202 Elche (Alicante, Spain)

Corresponding autor:

Dr. Javier Oltra-Cucarella, Dpto. Psicología de la Salud, Universidad Miguel Hernández de Elche, Avda de la Universidad s/n, Edificio Altamira, 03202 Elche (Alicante, Spain), e-mail address: joltra@umh.es

* Data used in preparation of this article were obtained from the Alzheimer's Disease Neuroimaging Initiative (ADNI) database (adni.loni.usc.edu). As such, the investigators within the ADNI contributed to the design and implementation of ADNI and/or provided data but did not participate in analysis or writing of this report. A complete listing of ADNI investigators can be found at: http://adni.loni.usc.edu/wpcontent/uploads/how to apply/ADNI_Acknowledgement_List.pdf

Declarations of interests: none 


\begin{abstract}
The linear regression-based Reliable Change Index (RCI) is widely used to identify memory impairments through longitudinal assessment. However, the minimum sample size required for estimates to be reliable has never been specified. Using the Alzheimer's Disease Neuroimaging Initiative data as true parameters, we run simulations for samples of size 10 to 1000 and analyzed the percentage of times the estimates are significant, their coverage rate, and the accuracy of the models including both the True Positive Rate (TPR) and the True Negative Rate (TNR). We compared the linear RCI with a logistic RCI for discrete, bounded scores. We found that the logistic RCI is more accurate than the linear RCI overall, with the linear RCI approximating the logistic RCI for samples of size 200 or greater. We provide an $\mathrm{R}$ code for researchers and clinicians to calculate the logistic RCI with samples smaller than 200.
\end{abstract}

Key-words: assessment, dementia, mild cognitive impairment, reliable change index, statistical models 


\section{Logistic versus linear regression-based Reliable Change Index: implications for clinical studies with diverse sample sizes}

Standard verbal memory tests are essential in the neuropsychological assessment of memory functioning, and are necessary to capture memory impairments in people with mild cognitive impairment (MCI) due to Alzheimer's disease (AD) who are at a greater risk of developing AD (Albert et al., 2011; Winblad et al., 2004). In the standard assessment of memory functioning, individuals are administered a verbal memory task and raw scores are compared against normative data obtained from a reference group (Strauss et al., 2006). However, performance on verbal memory tests can be interpreted using statistical techniques derived for serial assessment. These techniques, encompassed under the term Reliable Change Index (Duff, 2012), were developed to identify change in longitudinal assessment that exceeds normal variability (HintonBayre, 2011). Among several RCI methods that are available for the interpretation of significant change (Calamia et al., 2012; Duff, 2012), one of the most statistically developed technique is the standard regression-based $\mathrm{RCI}\left(\mathrm{RCI}_{\mathrm{RB}}\right)$. With the $\mathrm{RCI} \mathrm{I}_{\mathrm{RB}}$, a comparison group is used to predict scores on the second assessment using scores on the first assessment (Duff, 2012). In order to interpret whether reliable change has occurred, observed scores at the second assessment are subtracted from the expected scores based on the regression equation, and the discrepancy is standardized using the standard error of the regression equation (SEE).

The $\mathrm{RCI}_{\mathrm{RB}}$ has been applied to analyze memory impairments in different samples, including high school athletes (Brett et al., 2016), patients with epilepsy (Busch et al., 2015), traumatic brain injury (Metcalf et al., 2019), migraine (Roebuckspencer et al., 2007), cancer (Ouimet et al., 2009), HIV (Cysique et al., 2011), cardiac surgery (Sweet et al., 2008), schizophrenia (Roseberry \& Kristian Hill, 2014) or psychosis (Sánchez- 
Torres et al., 2018), dementia (O'Connell et al., 2019) or MCI (Campos-Magdaleno et al., 2017; Duff et al., 2017), older adults with total joint replacement (Scott et al., 2017), as well as healthy individuals of different ages (Bouman et al., 2015; Crockford et al., 2018; Elbin et al., 2019; Frerichs \& Tuokko, 2006; Gavett et al., 2015; Gonçalves et al., 2016; Raymond et al., 2006b; Salinsky et al., 2001; Schatz \& Ferris, 2013; Temkin et al., 1999; Van Der Elst et al., 2008), and non-demented older adults (Duff, 2014; Sánchez-Benavides et al., 2016).

As with any other statistical technique, assumptions about linear regression analyses must be met for the regression equation to be accurate. Assumptions of regression analysis are that the residuals (differences between obtained and predicted scores on the dependent variable -DV) are normally distributed around the predicted DV scores, are independent for each value of the predictor (Tabachnick \& Fidell, 2013), and that the variance of the residuals is the same for all values of the predictors, especially for small sample sizes (Williams et al., 2013). Following the Gauss-Markov theorem, even when residuals are not normally distributed, ordinary least squared parametric estimates are the best linear unbiased estimates (Williams et al., 2013). The violation of the assumption about normality of residuals affects significance tests and confidence interval of regression coefficients, even if they are still unbiased (Williams et al., 2013). This means that regression coefficients close to the real parameter might go undetected (increasing the false negative rate) if confidence intervals are too large (i.e., include the 0 ), or that regression coefficients that deviate from the real parameter might reach statistical significance (increasing the false positive rate), especially in small samples (Williams et al., 2013).

Heterogeneity in sample sizes is quite large in studies using the $\mathrm{RCI}_{\mathrm{RB}}$, ranging from samples equal or smaller than 30 (Metcalf et al., 2019; Nakhutina et al., 2010; 
Ouimet et al., 2009; Roebuckspencer et al., 2007; Roseberry \& Kristian Hill, 2014; Schatz \& Ferris, 2013; Sherman et al., 2003, 2003), to samples equal or smaller than 100 (Busch et al., 2015; Crockford et al., 2018; Elbin et al., 2019; Gonçalves et al., 2016; Hermann et al., 1996; Martin et al., 2002, 2006; Meekes et al., 2013, 2014; Raymond et al., 2006a, 2006b; Salinsky et al., 2001; Scott et al., 2017; Sweet et al., 2008), samples larger than 100 (Bouman et al., 2015; Campos-Magdaleno et al., 2017; Cysique et al., 2011; Duff, 2014; Duff et al., 2010; Frerichs \& Tuokko, 2006; Kashyap et al., 2014; Sánchez-Benavides et al., 2016; Sánchez-Torres et al., 2018; Temkin et al., 1999; Womble et al., 2016), and even samples larger than 500 (Brett et al., 2016; Gavett et al., 2015; Tombaugh, 2005; Van Der Elst et al., 2008). However, residuals have barely been tested or plotted, and thus the probability of using unreliable estimates is unknown. The aim of the present work was to analyze the sample size needed to increase the number of true positives and to reduce the number of false negatives to a minimum, in order to identify correctly individuals with objective longitudinal memory decline. As a further step, we provide an R code with an alternative method to model cognitive scores when analyzing reliable change with discrete, bounded scores from memory tests.

\section{Methods}

Data were obtained from the Alzheimer's Disease Neuroimaging Initiative (ADNI) database (adni.loni.usc.edu), launched in 2003 as a public-private partnership, led by Principal Investigator Michael W. Weiner, MD. The first ADNI period (ADNI1) was updated in the ADNIGO and ADNI2 grant periods. Information about magnetic resonance imaging, positron emission tomography, other biological markers and clinical and neuropsychological assessment are available for more than 1,000 normal controls, 
individuals with MCI, and individuals with mild dementia (Petersen et al., 2010) (www.adni-info.org). The ethical committee at each participating site approved the project. All ADNI participants provided written consent. All participants received physical and neurological examinations, screening laboratory tests, and provided blood samples for DNA and APOE testing. We used data from all participants: those labeled as cognitively normal or as having MCI at baseline. Cognitively normal participants had no memory complaints, Clinical Dementia Rating (Hughes et al., 1982) scale $=0$, MiniMental State Examination (Folstein et al., 1975) scores $\geq 24$, normal education-corrected Logical Memory subtest scores, and no significant impairments in activities of daily living. Participants with MCI met Petersen (1999) criteria: they had memory complaints, Clinical Dementia Rating (Hughes et al., 1982) scale $=0.5$, Mental State Examination (Folstein et al., 1975) scores $\geq 24$, education-corrected Logical Memory subtest scores equal or lower than 1.5 standard deviations below the mean of a normative sample, no significant impairments in activities of daily living, and did not meet criteria for dementia.

\section{Linear Regression-Based Relative Change Index (Linear RCI)}

Let $Y_{i}$ be the random variable representing the score obtained by individual $i, i=$ $1, \ldots, n$. We begin by assuming the distribution of $Y_{i}$ is normal with mean $\mu_{i}$ and variance $\sigma^{2}$, with $\mu_{i}=\boldsymbol{x}_{i}^{T} \boldsymbol{\beta}$, where $\boldsymbol{x}_{i}^{T}$ is the $i$-th row of the design matrix and $\boldsymbol{\beta}$ is the vector of regression coefficients. This is a standard multiple linear regression model,

with $\widehat{\boldsymbol{\beta}}=\left(\boldsymbol{X}^{\boldsymbol{T}} \boldsymbol{X}\right)^{-1} \boldsymbol{X}^{\boldsymbol{T}} \boldsymbol{y}$ and $\widehat{\sigma^{2}}=\frac{(\boldsymbol{y}-\boldsymbol{X} \widehat{\boldsymbol{\beta}})^{T}(\boldsymbol{y}-\boldsymbol{X} \widehat{\boldsymbol{\beta}})}{n-p}$ the well-known least squares estimators for $\boldsymbol{\beta}$ and $\sigma^{2}$, respectively, where $p$ is the dimension of the $\boldsymbol{\beta}$ vector. 
Crawford and Garthwaite (Crawford \& Garthwaite, 2007) refers to $\hat{\sigma}$ as the SEE. The Linear Regression-Based Reliable Change Index (Linear RCI) is given by

$$
\text { Linear RCI }=\frac{y_{i}-\hat{\mu}_{i}}{\hat{\sigma}}
$$

where $\hat{\mu}_{i}=\boldsymbol{x}_{i}^{T} \widehat{\boldsymbol{\beta}}$ is the predicted mean score for each individual (Crawford \& Garthwaite, 2007). Assuming the model is well fitted, the residuals are meant to follow a normal distribution. Since the Linear RCI is a standardised version of the raw residuals, for a well fitted model it is assumed to follow a standard normal distribution. Therefore, values that fall in the lower tail of the distribution are assumed to represent reliable decline. In this work, we used the $5 \%$ percentile (one-tailed) of the standard normal distribution (i.e., z-score $\leq-1.64$ ) as a threshold to detect reliable decline (Crawford \& Garthwaite, 2007). The effects of the terms in the model are assessed via $\mathrm{F}$ tests.

\section{Logistic Regression-Based Reliable Change Index (Logistic RCI)}

The response variable in this study, the Auditory Verbal learning Test Delayed Recall score (AVLT-DR) (Rey, 1964), is a discrete score that is bounded between zero and 15. Therefore, its nature is of a discrete proportion, and a sensible modelling approach would involve binomial generalized linear models (GLMs) and extensions. Here we propose a new RCI based on logistic regression: a binomial GLM with a logit link. Let $Y_{i}$ be the random variable representing the number of correct questions obtained by individual $i$. We may assume that the distribution of $Y_{i}$ is $\operatorname{Binomial}\left(m_{i}, \pi_{i}\right)$, where $m_{i}=15$ is the denominator of the distribution and $\pi_{i}$ is the probability of a 
question being correct for individual $i$. We model $\pi_{i}$ as a function of different predictors, in the logit (or log-odds) scale, i.e.

$$
\log \left(\frac{\pi_{i}}{1-\pi_{i}}\right)=\eta_{i}=\boldsymbol{x}_{i}^{T} \boldsymbol{\beta}
$$

where $\boldsymbol{x}_{i}^{T}$ is the $i$-th row of the design matrix and $\boldsymbol{\beta}$ is the vector of regression coefficients. Typically, the design matrix includes an intercept and the effects of baseline score, and may also include other covariates such as age, gender and education level. By fitting a logistic regression model, we are able to estimate the regression coefficients and the linear predictor $\hat{\eta}_{i}=\boldsymbol{x}_{i}^{T} \widehat{\boldsymbol{\beta}}$. Consequently, the probability of a question being correct would be represented as $\hat{\pi}_{i}=\frac{e^{\hat{\eta}_{i}}}{1+e^{\hat{\eta}_{i}}}$.

The Logistic Regression-Based Relative Change Index (Logistic RCI) is based on the Anscombe residuals for generalized linear models, combined with the correction proposed by Cox and Snell (1968) to stabilise the asymptotic variance of their distribution. We may write it as

$$
\text { Logistic RCI }=\sqrt{m_{i}} \frac{\delta\left(\frac{y_{i}}{m_{i}}\right)-\delta\left(\hat{\pi}_{i}-\frac{1-2 \hat{\pi}_{i}}{6 m_{i}}\right)}{\left\{\hat{\pi}_{i}\left(1-\hat{\pi}_{i}\right)\right\}^{-\frac{1}{6}}}
$$

, where $\delta(x)=\int_{0}^{x}\{t(1-t)\}^{-\frac{1}{3}} d t$ is the incomplete beta function. Asymptotically, the Logistic RCI has a normal distribution with mean zero and variance that depends on $\hat{\pi}_{i}$. We may standardise it to obtain $z$-scores by dividing the logistic RCI by its observed standard deviation, therefore yielding an asymptotic $N(0,1)$ distribution. Again, we used the $5 \%$ percentile of the standard normal distribution as a threshold to detect reliable decline (i.e. $\mathrm{z}$-score $\leq-1.64$ ). 
We fit the binomial GLMs allowing $\operatorname{Var}\left(Y_{i}\right)=\phi m_{i} \pi_{i}\left(1-\pi_{i}\right)$, and estimate $\phi$ via a quasi-likelihood approach using the Pearson residuals, thus allowing for a more flexible variance function that accommodates extra variability, should that be present in the data. Finally, we assess the significance of the model effects using F tests, since the dispersion parameter $\phi$ has been estimated.

\section{Assessing Model Goodness-of-fit and Normality of the RCI}

To assess model overall goodness-of-fit, we produced half-normal plots with a simulated envelope for the studentised residuals for the linear RCI and deviance residuals for the logistic RCI. This is obtained by plotting the ordered absolute values of the residuals versus the expected order statistics of a half-normal distribution (Moral et al., 2017). By simulating data from the fitted models, refitting the models and obtaining the ordered absolute values of the residuals, we may obtain an envelope by computing the $97.5 \%$ and $2.5 \%$ percentile for each order statistics. The envelope is such that for a well-fitting model, we would expect most points to lie within it. We assessed the normality of the linear RCI and the logistic RCI by producing the half-normal plot with a simulated envelope and counting the number of points that fell outside of the envelope (the more points that fall outside, the bigger the departure from normality).

\section{Simulation Study}

We began by fitting a linear and a logistic regression model to the ADNI dataset including baseline score, age, education and gender in the linear predictor, and treated the estimated parameters as the true population parameters (see Table 1). Then, we simulated a population of 1 million individuals based on these estimated parameters and 
predefined distributions for baseline score, age, education and gender that are similar to the ones observed in the ADNI dataset. After that, we drew 1000 samples of sizes 10 , $20,30,40,50,100,150,200,250,500,750$ and 1000 from the simulated dataset, refitted the linear and logistic regression models to each sample, and calculated the linear and the logistic RCI. We then identified individuals with z-score $\leq 1.64$ as showing reliable decline.

Finally, we assessed (i) the significance of the effects in the linear predictor, (ii) the normality of the regression-based RCI (based on the half-normal plot with a simulation envelope) (Moral et al., 2017), (iii) the percentage of points outside the envelope of the half-normal plot of the residuals (as a measure of overall goodness-offit), (iv) the percentage coverage of the $90 \%, 95 \%$ and $99 \%$ confidence intervals for each true parameter value, and (v) the accuracy (true positive and true negative rates) for detecting reliable decline at different thresholds.

For the logistic RCI, the baseline scores were simulated from a $\operatorname{Beta}(1.59,1.36)$ distribution. This distribution was obtained by fitting a beta model to 15 minus the baseline scores observed in the ADNI dataset. The age variable was simulated from a $\mathrm{N}(73.41,46.78)$ distribution, which was obtained by fitting a normal model to the observed ages in the ADNI dataset. The gender variable was simulated from a Bernoulli(0.5) distribution, so that approximately half of the individuals were male and half female. Finally, the education level variable was simulated from a discrete uniform distribution, ranging from 4 to 20 , the range of the education variable in the ADNI dataset. All simulations and visualisations were produced using R (R Core Team, 2021), and all associated code is made available at https://github.com/rafamoral/LogisticRCI. 


\section{Results}

Looking at the percentage of times the $\mathrm{F}$ test was significant for each effect (see Figure 1), linear and logistic models yielded very similar results. For the baseline score, even with a sample size as small as 20 , we already observed significance (associated pvalue less than 0.05 ) for $100 \%$ of the samples. The effect of baseline score, which is the slope of the curve, is the largest in magnitude (see Table 1), and therefore even with a small sample size it is not difficult to obtain a significant estimate. Education, however, is of a smaller magnitude and significance was attained for $100 \%$ of samples of size 150 or larger. When looking at the effects of age and gender, it seems that it is very difficult for the method to obtain significant estimates, even with a sample as large as 1000, especially for the effect of gender.

When studying the coverage of the $90 \%, 95 \%$ and $99 \%$ confidence intervals (Supplementary Material), again we observed very similar results between the linear and logistic models. The coverage for the intercept, baseline and age effects was very close to the nominal coverage rate for samples as small as 30 . For the gender effect, coverage was systematically above the nominal rate. This is because the gender effect is typically associated with a large standard error, and therefore the confidence intervals are inflated. The education level effect, on the other hand, presented coverage systematically below the nominal rate, although very close to it.

The distribution of the linear and logistic regression based RCI was considered to be normal for most simulated datasets, at very similar rates, based on the half-normal plot with a simulated envelope (Supplementary Material). Model goodness-of-fit, however, was systematically better for the logistic model when compared to the linear model, although as discussed above, inferential power seemed to be very similar for both modelling approaches. 
When attempting to identify reliable change, the true positive rates (TPR) for the logistic RCI are systematically greater than the TPR for the linear RCI for smaller sample sizes (200 or less, see Fig. 2, top-left panel). The true negative rates (TNR), however, are very similar for both approaches, and close to $100 \%$, although the TNR for the linear RCI is slightly lower. It becomes clear that the overall accuracy (bottom panels of Fig. 2) is dictated by the TPR in this case, and the logistic RCI presents better performance overall.

In order to analyze whether the results might be biased because of including both cognitively normal and participants with MCI in the sample used to obtain the estimates for the simulations, we rerun the analyses using only cognitively normal participants. The results remained unchanged (data not shown).

\section{Discussion}

The present work aimed to analyze the sample size needed to obtain reliable estimates when assessing memory decline with the standard linear RCI. Additionally, we analyzed through simulation whether the identification of reliable decline was as accurate (true positives and true negatives) when using a linear model as when using a logistic model. We used the AVLT delayed recall scores, which have been reported as a test of high accuracy (Ozer et al., 2016). The simulation showed that both models give similar results for samples sizes of 200 or greater, with the logistic RCI presenting better performance with smaller sample sizes.

The main implications raise when evaluating the accuracy in terms of sensitivity (detection of true positives) and specificity (detection of true negatives). For samples smaller than 200, which are quite frequent in the literature of clinical research in older 
adults and dementia, the TPR for the logistic RCI are systematically greater than the TPR for the linear RCI, whereas the TNR for the linear RCI are only slightly lower. In other words, this implies that traditionally estimated linear RCI has higher rates of both false positives (individuals incorrectly identified as having reliable decline) and false negatives (individuals incorrectly identified as not having reliable decline). For the subject of false positives, Klekociuk et al. (2014) stated that the rate of recovery observed in MCI indicates that existing MCI diagnostic criteria comprise an unacceptably high rate of false positive diagnoses and lack adequate sensitivity and specificity. Edmonds et al. (2015) reported similar concerns as their results showed that a significant proportion of individuals in the ADNI/MCI sample are cognitively normal if more detailed testing is taken into account, and that a subset of individuals from the cluster-derived normal group could be at risk of developing MCI (thus uncovering the relevance of potential false negative cases).

It has been highlighted in the literature that the potential impact of false negatives has remained largely ignored (Vadillo et al., 2016), and the over-interpretation of null results is even more dangerous than the prevalence of false positives in some areas of research, since null results (1) are inherently ambiguous, (2) they are silent about the amount of support for the null hypothesis, and (3) they are surprisingly easy to obtain by mere statistical artefacts (e.g., using a small sample or a noisy measure can suffice to produce a false negative). As Edmonds et al. (2016) state, the impact of "missed" cases of MCI because of being wrongly discarded as healthy has a direct impact in clinical practice, but also in research studies and clinical trials targeting prodromal AD.

Knowing the impact that high FNR have on clinical practice, the linear RCI seems to be unreliable for small sample sizes. Using a logistic RCI that decreased the FNR would be extremely helpful in the sense that less individuals would miss 
opportunities for intervention (in the form of cognitive rehabilitation) and they would subsequently engage in potentially beneficial treatments from early stages, with clinicians being more confident in the type of recommendations provided to them and their families. In addition, early interventions targeting risk factors by encouraging both physical and cognitive activities would reach a higher percentage of population. Where necessary, compensatory strategies in the form of external aids would be applied at earlier stages, as well as referrals to other professionals for further assessment (Edmonds et al., 2016). This positive scenario of higher accuracy in the detection of reliable decline can be achieved if traditional reliance on linear models for RCI estimation is overcome. Our results suggest that for typical sample sizes used in many clinical studies (an average $\mathrm{n}<200$ individuals), a logistic model can more accurately both identify actual clinical cases and discard healthy individuals, regardless of other intervening factors such as age or gender, and with the impact of education being more clear with higher sample sizes.

Additionally, our results suggest an alternative way of improving the accuracy of the RCI. Researchers and clinicians may be less conservative when setting up the threshold to identify reliable change. This yields improved TPR at the expense of a smaller TNR. As shown in the middle panels in figure 2, when changing the threshold from -1.64 to -1.28 the TPR is above $95 \%$ for the logistic RCI even for samples as small as 10 , rising to almost $100 \%$ for samples of size 100 and larger, when compared to a TPR of around $85 \%$ when using the more conservative threshold. This improvement is obtained at the expense of lowering the TNR and overall accuracy from close to $100 \%$ to around $98 \%$. With regards to the linear RCI, lowering the threshold to -1.28 rises the TPR to values higher than $90 \%$ for samples of size 50 while maintaining the TNR above 
$95 \%$. For small sample sizes, the linear model seems to be unreliable for either threshold.

However, caution is needed when modifying the threshold used to identify reliable decline. Lowering the threshold to -1.28 will allow classifying more observations as showing reliable decline, which will increase the number of true positives, but also the number of false positives. If we keep relaxing the lower bound, say to -1.04 (right-hand panels on Fig. 2) we see that the improvement in TPR is not that different from when using -1.28 , however the TNR and overall accuracy now fall to around $95 \%$. If it is more important to identify those individuals who present reliable decline, at the expense of obtaining a few more false positives, then we recommend relaxing the lower bound to a value greater than -1.64 . We are not, however, advocating for a hard threshold of -1.28 . We are simply pointing out that if we assume that in the population the $5 \%$ lower percentile are representative of reliable decline, when analysing smaller samples it could be a good idea to look at a higher percentile of the samples to identify reliable change (e.g., the $10^{\text {th }}$ percentile). This would increase the TPR at a small expense of lowering the TNR only a little. This is in line with the common use of normative data to interpret performance on neuropsychological tests (e.g., in MCI research), where the $7^{\text {th }}$ percentile $(z=-1.5)$ is used to identify low scores.

The implications of our results is that, based on the higher FNR associated with the linear RCI, several studies with and small sample sizes seem unreliable to identify reliable decline (Busch et al., 2015; Crockford et al., 2018; Elbin et al., 2019; Gonçalves et al., 2016; Hermann et al., 1996; Martin et al., 2002, 2006; Meekes et al., 2013, 2014; Metcalf et al., 2019; Nakhutina et al., 2010; Ouimet et al., 2009; Raymond et al., 2006a, 2006b; Roebuckspencer et al., 2007; Roseberry \& Kristian Hill, 2014; Salinsky et al., 2001; Schatz \& Ferris, 2013; Scott et al., 2017; Sherman et al., 2003, 2003; Sweet et al., 
2008). There is no way to know whether the estimates reported in these studies are false, as it is not possible to gather the real parameters in the population, but the results from our simulation suggesting that the probability of having left unidentified a large proportion of individuals with cognitive impairment is high raises concerns about their conclusions. Replication studies with sample of sizes larger than 200 are needed.

This study comprises limitations as it involves a modest approach to detection of changes based on discrete scores of an episodic memory test. Klekociuk et al. (2014) highlighted the importance of using comprehensive test batteries to enhance sensitivity and specificity in MCI classification by including both memory and non-memory assessments. In addition, Blanco-Campal et al. (2019) suggested that it would be interesting to identify cases with a raw score below or above the standard cut-point but whose qualitative performance may point in the opposite direction (e.g., score above the cut-point with indications of decline of clinical relevance). In any case, our study has shown that the application of a logistic RCI can increase the accuracy of identifying reliable decline and improve true positive and true negative rates, and has provided a pathway to identify the relevance of incorporating moderate to high sample sizes in future clinical studies. It is reasonable to assume that this same model will show more accurate results when data from multiple sources, both memory and non-memory tests, are taken into consideration. Additional research is needed to determine whether logistic models using additional scores from multiple tests and diverse clinical samples can improve the estimation of an accurate RCI further. 
Acknowledgements

Data collection and sharing for this project was funded by the Alzheimer's Disease Neuroimaging Initiative (ADNI) (National Institutes of Health Grant U01 AG024904) and DOD ADNI (Department of Defense award number W81XWH-12-2-0012). ADNI is funded by the National Institute on Aging, the National Institute of Biomedical Imaging and Bioengineering, and through generous contributions from the following: AbbVie, Alzheimer's Association; Alzheimer's Drug Discovery Foundation; Araclon Biotech; BioClinica, Inc.; Biogen; Bristol-Myers Squibb Company; CereSpir, Inc.; Cogstate; Eisai Inc.; Elan Pharmaceuticals, Inc.; Eli Lilly and Company; EuroImmun; F. Hoffmann-La Roche Ltd and its affiliated company Genentech, Inc.; Fujirebio; GE Healthcare; IXICO Ltd.; Janssen Alzheimer Immunotherapy Research \& Development, LLC.; Johnson \& Johnson Pharmaceutical Research \& Development LLC.; Lumosity; Lundbeck; Merck \& Co., Inc.; Meso Scale Diagnostics, LLC.; NeuroRx Research; Neurotrack Technologies; Novartis Pharmaceuticals Corporation; Pfizer Inc.; Piramal Imaging; Servier; Takeda Pharmaceutical Company; and Transition Therapeutics. The Canadian Institutes of Health Research is providing funds to support ADNI clinical sites in Canada. Private sector contributions are facilitated by the Foundation for the National Institutes of Health (www.fnih.org). The grantee organization is the Northern California Institute for Research and Education, and the study is coordinated by the Alzheimer's Therapeutic Research Institute at the University of Southern California. ADNI data are disseminated by the Laboratory for Neuro Imaging at the University of Southern California. ADNI had no involvement in study design; in the analysis and interpretation of data; in the writing of the report; and in the decision to submit the article for publication. 


\section{References}

Albert, M. S., DeKosky, S. T., Dickson, D., Dubois, B., Feldman, H. H., Fox, N. C., Gamst, A., Holtzman, D. M., Jagust, W. J., Petersen, R. C., Snyder, P. J., Carrillo, M. C., Thies, B., \& Phelps, C. H. (2011). The diagnosis of mild cognitive impairment due to Alzheimer's disease: Recommendations from the National Institute on Aging-Alzheimer's Association workgroups on diagnostic guidelines for Alzheimer's disease. Alzheimer's \& Dementia, 7(3), 270-279. https://doi.org/10.1016/j.jalz.2011.03.008

Blanco-Campal, A., Diaz-Orueta, U., Navarro-Prados, A. B., Burke, T., Libon, D. J., \& Lamar, M. (2019). Features and psychometric properties of the Montreal Cognitive Assessment: Review and proposal of a process-based approach version (MoCA-PA). Applied Neuropsychology: Adult, 1-15. https://doi.org/10.1080/23279095.2019.1681996

Bouman, Z., Hendriks, M. P. H., Aldenkamp, A. P., \& Kessels, R. P. C. (2015). Temporal Stability of the Dutch Version of the Wechsler Memory ScaleFourth Edition (WMS-IV-NL). The Clinical Neuropsychologist, 29(sup1), 3046. https://doi.org/10.1080/13854046.2015.1137354

Brett, B. L., Smyk, N., Solomon, G., Baughman, B. C., \& Schatz, P. (2016). Long-term Stability and Reliability of Baseline Cognitive Assessments in High School Athletes Using ImPACT at 1-, 2-, and 3-year Test-Retest Intervals. Archives of Clinical Neuropsychology, acn;acw055v1. https://doi.org/10.1093/arclin/acw055

Busch, R. M., Lineweaver, T. T., Ferguson, L., \& Haut, J. S. (2015). Reliable change indices and standardized regression-based change score norms for evaluating neuropsychological change in children with epilepsy. Epilepsy \& Behavior, 47, 45-54. https://doi.org/10.1016/j.yebeh.2015.04.052 
Calamia, M., Markon, K., \& Tranel, D. (2012). Scoring Higher the Second Time Around: Meta-Analyses of Practice Effects in Neuropsychological Assessment. The Clinical Neuropsychologist, 26(4), 543-570. https://doi.org/10.1080/13854046.2012.680913

Campos-Magdaleno, M., Facal, D., Lojo-Seoane, C., Pereiro, A. X., \& Juncos-Rabadán, O. (2017). Longitudinal Assessment of Verbal Learning and Memory in Amnestic Mild Cognitive Impairment: Practice Effects and Meaningful Changes. Frontiers in Psychology, 8. https://doi.org/10.3389/fpsyg.2017.01231

Cox, D. R., \& Snell, E. J. (1968). A General Definition of Residuals. Journal of the Royal Statistical Society. Series B (Methodological), 30(2), 248-275.

Crawford, J. R., \& Garthwaite, P. H. (2007). Using regression equations built from summary data in the neuropsychological assessment of the individual case. Neuropsychology, 21(5), 611-620. https://doi.org/10.1037/0894-4105.21.5.611

Crockford, C., Newton, J., Lonergan, K., Madden, C., Mays, I., O’Sullivan, M., Costello, E., Pinto-Grau, M., Vajda, A., Heverin, M., Pender, N., Al-Chalabi, A., Hardiman, O., \& Abrahams, S. (2018). Measuring reliable change in cognition using the Edinburgh Cognitive and Behavioural ALS Screen (ECAS). Amyotrophic Lateral Sclerosis and Frontotemporal Degeneration, 19(1-2), 6573. https://doi.org/10.1080/21678421.2017.1407794

Cysique, L. A., Franklin, D., Abramson, I., Ellis, R. J., Letendre, S., Collier, A., Clifford, D., Gelman, B., McArthur, J., Morgello, S., Simpson, D., McCutchan, J. A., Grant, I., Heaton, R. K., the CHARTER group, \& the HNRC group. (2011). Normative data and validation of a regression based summary score for assessing meaningful neuropsychological change. Journal of Clinical and 
Experimental Neuropsychology, 33(5), 505-522.

https://doi.org/10.1080/13803395.2010.535504

Duff, K. (2012). Evidence-Based Indicators of Neuropsychological Change in the Individual Patient: Relevant Concepts and Methods. Archives of Clinical Neuropsychology, 27(3), 248-261. https://doi.org/10.1093/arclin/acr120

Duff, K. (2014). One-Week Practice Effects in Older Adults: Tools for Assessing Cognitive Change. The Clinical Neuropsychologist, 28(5), 714-725. https://doi.org/10.1080/13854046.2014.920923

Duff, K., Atkinson, T. J., Suhrie, K. R., Dalley, B. C. A., Schaefer, S. Y., \& Hammers, D. B. (2017). Short-term practice effects in mild cognitive impairment: Evaluating different methods of change. Journal of Clinical and Experimental Neuropsychology, 39(4), 396-407. https://doi.org/10.1080/13803395.2016.1230596

Duff, K., Beglinger, L. J., Moser, D. J., \& Paulsen, J. S. (2010). Predicting cognitive change within domains. The Clinical Neuropsychologist, 24(5), 779-792. https://doi.org/10.1080/13854041003627795

Edmonds, E. C., Delano-Wood, L., Clark, L. R., Jak, A. J., Nation, D. A., McDonald, C. R., Libon, D. J., Au, R., Galasko, D., Salmon, D. P., \& Bondi, M. W. (2015). Susceptibility of the conventional criteria for mild cognitive impairment to falsepositive diagnostic errors. Alzheimer's \& Dementia, 11(4), 415-424. https://doi.org/10.1016/j.jalz.2014.03.005

Edmonds, E. C., Delano-Wood, L., Jak, A. J., Galasko, D. R., Salmon, D. P., \& Bondi, M. W. (2016). "Missed” Mild Cognitive Impairment: High False-Negative Error Rate Based on Conventional Diagnostic Criteria. Journal of Alzheimer's Disease, 52(2), 685-691. https://doi.org/10.3233/JAD-150986 
Elbin, R. J., Fazio-Sumrok, V., Anderson, M. N., D’Amico, N. R., Said, A., Grossel, A., Schatz, P., Lipinski, D., \& Womble, M. (2019). Evaluating the suitability of the Immediate Post-Concussion Assessment and Cognitive Testing (ImPACT) computerized neurocognitive battery for short-term, serial assessment of neurocognitive functioning. Journal of Clinical Neuroscience, 62, 138-141. https://doi.org/10.1016/j.jocn.2018.11.041

Folstein, M. F., Folstein, S. E., \& McHugh, P. R. (1975). “Mini-mental state". A practical method for grading the cognitive state of patients for the clinician. Journal of Psychiatry Research, 12(3), 189-198. https://doi.org/10.1016/00223956(75)90026-6

Frerichs, R. J., \& Tuokko, H. A. (2006). Reliable change scores and their relation to perceived change in memory: Implications for the diagnosis of mild cognitive impairment. Arch. Clin. Neuropsychol., 21(1), 109-115. https://doi.org/10.1016/j.acn.2005.08.003

Gavett, B. E., Ashendorf, L., \& Gurnani, A. S. (2015). Reliable Change on Neuropsychological Tests in the Uniform Data Set. Journal of the International Neuropsychological Society, 21(7), 558-567. https://doi.org/10.1017/S1355617715000582

Gonçalves, M. M., Pinho, M. S., \& Simões, M. R. (2016). Test-retest reliability analysis of the Cambridge Neuropsychological Automated Tests for the assessment of dementia in older people living in retirement homes. Applied Neuropsychology: Adult, 23(4), 251-263. https://doi.org/10.1080/23279095.2015.1053889

Hermann, B. P., Seidenberg, M., Schoenfeld, J., Peterson, J., Leveroni, C., \& Wyler, A. R. (1996). Empirical Techniques for Determining the Reliability, Magnitude, 
and Pattern of Neuropsychological Change After Epilepsy Surgery. Epilepsia, 37(10), 942-950. https://doi.org/10.1111/j.1528-1157.1996.tb00531.x

Hinton-Bayre, A. D. (2011). Specificity of Reliable Change Models and Review of the Within-subjects Standard Deviation as an Error Term. Archives of Clinical Neuropsychology, 26(1), 67-75. https://doi.org/10.1093/arclin/acq087

Hughes, C. P., Berg, L., Danziger, W. L., Coben, L. A., \& Martin, R. L. (1982). A new clinical scale for the staging of dementia. British Journal of Psychiatry, 140(6), 566-572. https://doi.org/10.1192/bjp.140.6.566

Kashyap, M., Belleville, S., Mulsant, B. H., Hilmer, S. N., Paquette, A., Tu, L. M., \& Tannenbaum, C. (2014). Methodological Challenges in Determining Longitudinal Associations Between Anticholinergic Drug Use and Incident Cognitive Decline. Journal of the American Geriatrics Society, 62(2), 336-341. https://doi.org/10.1111/jgs.12632

Klekociuk, S. Z., Summers, J. J., Vickers, J. C., \& Summers, M. J. (2014). Reducing false positive diagnoses in mild cognitive impairment: The importance of comprehensive neuropsychological assessment. European Journal of Neurology, 21(10), 1330-e83. https://doi.org/10.1111/ene.12488

Martin, R., Griffith, H. R., Sawrie, S., Knowlton, R., \& Faught, E. (2006). Determining empirically based self-reported cognitive change: Development of reliable change indices and standardized regression-based change norms for the multiple abilities self-report questionnaire in an epilepsy sample. Epilepsy \& Behavior, 8(1), 239-245. https://doi.org/10.1016/j.yebeh.2005.10.004

Martin, R., Sawrie, S., Gilliam, F., Mackey, M., Faught, E., Knowlton, R., \& Kuzniekcy, R. (2002). Determining Reliable Cognitive Change after Epilepsy Surgery: Development of Reliable Change Indices and Standardized Regression- 
based Change Norms for the WMS-III and WAIS-III. Epilepsia, 43(12), 15511558. https://doi.org/10.1046/j.1528-1157.2002.23602.x

Meekes, J., Braams, O. B., Braun, K. P. J., Jennekens-Schinkel, A., van Rijen, P. C., Alpherts, W. C. J., Hendriks, M. P. H., \& van Nieuwenhuizen, O. (2014). Visual memory after epilepsy surgery in children: A standardized regression-based analysis of group and individual outcomes. Epilepsy \& Behavior, 36, 57-67. https://doi.org/10.1016/j.yebeh.2014.04.016

Meekes, J., Braams, O., Braun, K. P. J., Jennekens-Schinkel, A., \& van Nieuwenhuizen, O. (2013). Verbal memory after epilepsy surgery in childhood. Epilepsy Research, 107(1-2), 146-155. https://doi.org/10.1016/j.eplepsyres.2013.08.017

Metcalf, K., Sabaz, M., Daher, M., \& Simpson, G. (2019). Measuring reliable change in traumatic brain injury (TBI): The pitfalls of using readily available formulae. Applied Neuropsychology: Adult, 1-10. https://doi.org/10.1080/23279095.2018.1559166

Moral, R. A., Hinde, J., \& Demétrio, C. G. B. (2017). Half-Normal Plots and Overdispersed Models in R: The hnp Package. Journal of Statistical Software, 81(1), 1-23. https://doi.org/10.18637/jss.v081.i10

Nakhutina, L., Pramataris, P., Morrison, C., Devinsky, O., \& Barr, W. B. (2010). Reliable Change Indices and Regression-Based Measures for the Rey-Osterreith Complex Figure Test in Partial Epilepsy Patients. The Clinical Neuropsychologist, 24(1), 38-44. https://doi.org/10.1080/13854040902960091

O’Connell, M. E., Gould, B., Ursenbach, J., Enright, J., \& Morgan, D. G. (2019). Reliable change and minimum clinically important difference (MCID) of the Repeatable Battery for the Assessment of Neuropsychology Status (RBANS) in a heterogeneous dementia sample: Support for reliable change methods but not 
the MCID. Applied Neuropsychology: Adult, 26(3), 268-274.

https://doi.org/10.1080/23279095.2017.1413575

Ouimet, L. A., Stewart, A., Collins, B., Schindler, D., \& Bielajew, C. (2009). Measuring neuropsychological change following breast cancer treatment: An analysis of statistical models. Journal of Clinical and Experimental Neuropsychology, 31(1), 73-89. https://doi.org/10.1080/13803390801992725

Ozer, S., Young, J., Champ, C., \& Burke, M. (2016). A systematic review of the diagnostic test accuracy of brief cognitive tests to detect amnestic mild cognitive impairment: A systematic review of the DTA of brief cognitive tests to detect aMCI. International Journal of Geriatric Psychiatry, 31(11), 1139-1150. https://doi.org/10.1002/gps.4444

Petersen, R., Aisen, P. S., Beckett, L. A., Donohue, M. C., Gamst, A. C., Harvey, D. J., Jack, C. R., Jagust, W. J., Shaw, L. M., Toga, A. W., Trojanowski, J. Q., \& Weiner, M. W. (2010). Alzheimer's Disease Neuroimaging Initiative (ADNI): Clinical characterization. Neurology, 74(3), 201-209. https://doi.org/10.1212/WNL.0b013e3181cb3e25

Petersen, R., Smith, G., Waring, S., Ivnik, R., Tangalos, T., \& Kokmen, E. (1999). Mild cognitive impairment: Clinical characterization and outcome. Archives of Neurology, 56(3), 303-308. https://doi.org/10.1001/archneur.56.3.303

Raymond, P. D., Hinton-Bayre, A. D., Radel, M., Ray, M. J., \& Marsh, N. A. (2006a). Assessment of statistical change criteria used to define significant change in neuropsychological test performance following cardiac surgery. European Journal of Cardio-Thoracic Surgery, 29(1), 82-88.

https://doi.org/10.1016/j.ejcts.2005.10.016 
Raymond, P. D., Hinton-Bayre, A. D., Radel, M., Ray, M. J., \& Marsh, N. A. (2006b). Test-Retest Norms and Reliable Change Indices for the MicroCog Battery in a Healthy Community Population Over 50 Years of Age. The Clinical Neuropsychologist, 20(2), 261-270. https://doi.org/10.1080/13854040590947416

Rey, A. (1964). L'Examen Clinique en Psychologie. Presses Universitaires de France. Roebuckspencer, T., Sun, W., Cernich, A., Farmer, K., \& Bleiberg, J. (2007). Assessing change with the Automated Neuropsychological Assessment Metrics (ANAM): Issues and challenges 录. Archives of Clinical Neuropsychology, 22, 79-87. https://doi.org/10.1016/j.acn.2006.10.011

Roseberry, J. E., \& Kristian Hill, S. (2014). Limited practice effects and evaluation of expectation for change: MATRICS Consensus Cognitive Battery. Schizophrenia Research, 159(1), 188-192. https://doi.org/10.1016/j.schres.2014.08.004

Salinsky, M. C., Storzbach, D., Dodrill, C. B., \& Binder, L. M. (2001). Test-retest bias, reliability, and regression equations for neuropsychological measures repeated over a 12-16-week period. Journal of the International Neuropsychological Society: JINS, 7(5), 597-605.

Sánchez-Benavides, G., Peña-Casanova, J., Casals-Coll, M., Gramunt, N., Manero, R. M., Puig-Pijoan, A., Aguilar, M., Robles, A., Antúnez, C., Frank-García, A., Fernández-Martínez, M., Blesa, R., NEURONORMA Study Team, Oliva, R., Gómez-Ansón, B., Monte, G., Alayrach, E., Sainz, A., Caprile, C., ... Ribas, R. (2016). One-Year Reference Norms of Cognitive Change in Spanish Old Adults: Data from the NEURONORMA Sample. Archives of Clinical Neuropsychology, 31(4), 378-388. https://doi.org/10.1093/arclin/acw018 
Sánchez-Torres, A. M., Moreno-Izco, L., Lorente-Omeñaca, R., Cabrera, B., Lobo, A., González-Pinto, A. M., Merchán-Naranjo, J., Corripio, I., Vieta, E., de la Serna, E., Butjosa, A., Contreras, F., Sarró, S., Mezquida, G., Ribeiro, M., Bernardo, M., \& Cuesta, M. J. (2018). Individual trajectories of cognitive performance in first episode psychosis: A 2-year follow-up study. European Archives of Psychiatry and Clinical Neuroscience, 268(7), 699-711.

https://doi.org/10.1007/s00406-017-0857-z

Schatz, P., \& Ferris, C. S. (2013). One-Month Test-Retest Reliability of the ImPACT Test Battery. Archives of Clinical Neuropsychology, 28(5), 499-504. https://doi.org/10.1093/arclin/act034

Scott, J. E., Mathias, J. L., Kneebone, A. C., \& Krishnan, J. (2017). Postoperative cognitive dysfunction and its relationship to cognitive reserve in elderly total joint replacement patients. Journal of Clinical and Experimental Neuropsychology, 39(5), 459-472. https://doi.org/10.1080/13803395.2016.1233940

Sherman, E. M. S., Slick, D. J., Connolly, M. B., Steinbok, P., Martin, R., Strauss, E., Chelune, G. J., \& Farrell, K. (2003). Reexamining the effects of epilepsy surgery on IQ in children: Use of regression-based change scores. Journal of the International Neuropsychological Society, 9(6), 879-886. https://doi.org/10.1017/S1355617703960085

Strauss, E. H., Sherman, E. H. S., \& Spreen, O. (2006). A Compendium of Neuropsychological Tests. Administration, Norms and Comments. Oxford University Press.

Sweet, J. J., Finnin, E., Wolfe, P. L., Beaumont, J. L., Hahn, E., Marymont, J., Sanborn, T., \& Rosengart, T. K. (2008). Absence of Cognitive Decline One Year After 
Coronary Bypass Surgery: Comparison to Nonsurgical and Healthy Controls. The Annals of Thoracic Surgery, 85(5), 1571-1578.

https://doi.org/10.1016/j.athoracsur.2008.01.090

Tabachnick, B. G., \& Fidell, L. S. (2013). Using Multivariate Statistics. (Sixth Ed.). Pearson Education Inc.

Temkin, N. R., Heaton, R. K., Grant, I., \& Dikmen, S. S. (1999). Detecting significant change in neuropsychological test performance: A comparison of four models. Journal of the International Neuropsychological Society: JINS, 5(4), 357-369.

Tombaugh, T. (2005). Test-retest reliable coefficients and 5-year change scores for the MMSE and 3MS. Archives of Clinical Neuropsychology, 20(4), 485-503. https://doi.org/10.1016/j.acn.2004.11.004

Vadillo, M. A., Konstantinidis, E., \& Shanks, D. R. (2016). Underpowered samples, false negatives, and unconscious learning. Psychonomic Bulletin \& Review, 23(1), 87-102. https://doi.org/10.3758/s13423-015-0892-6

Van Der Elst, W., Van Boxtel, M. P. J., Van Breukelen, G. J. P., \& Jolles, J. (2008). Detecting the significance of changes in performance on the Stroop Color-Word Test, Rey's Verbal Learning Test, and the Letter Digit Substitution Test: The regression-based change approach. Journal of the International Neuropsychological Society, 14(1), 71-80. https://doi.org/10.1017/S1355617708080028

Williams, M. N., Gómez Grajales, C. A., \& Kurkiewicz, D. (2013). Assumptions of Multiple Regression: Correcting Two Misconceptions. Practical Assessment, Research \& Evaluation, 18(11), 1-14.

Winblad, B., Palmer, K., Kivipelto, M., Jelic, V., Fratiglioni, L., Wahlund, L.-O., Nordberg, A., Bäckman, L., Albert, M., Almkvist, O., Arai, H., Basun, H., 
Blennow, K., de Leon, M., DeCarli, C., Erkinjuntti, T., Giacobini, E., Graff, C., Hardy, J., ... Petersen, R. C. (2004). Mild cognitive impairment-beyond controversies, towards a consensus: Report of the International Working Group on Mild Cognitive Impairment. Journal of Internal Medicine, 256(3), 240-246. https://doi.org/10.1111/j.1365-2796.2004.01380.x

Womble, M. N., Reynolds, E., Schatz, P., Shah, K. M., \& Kontos, A. P. (2016). TestRetest Reliability of Computerized Neurocognitive Testing in Youth Ice Hockey Players. Archives of Clinical Neuropsychology, 31(4), 305-312.

https://doi.org/10.1093/arclin/acw011 


\section{Figure Captions}

Figure 1: Percentage of times the $p$-value associated to the F-test was less than 0.05 , i.e. indicated a significant effect, for age, the baseline score, education and gender, for the linear and logistic regression models fitted to the simulated datasets. We drew 1,000 samples of sizes 10, 20, 30, 40, 50, 100, 150, $200,250,500,750$ and 1,000 at random from a simulated population comprising of one million individuals. True parameter values are indicated in Table1.

Figure 2: True positive rates, true negative rates and overall accuracy for identifying reliable decline for the linear and logistic reliable change index, using different threshold values: -1.64 corresponding to the $5 \%$ percentile of the standard normal distribution, -1.28 corresponding to the $10 \%$ percentile and -1.04 corresponding to the $15 \%$ percentile. Reliable decline is identified when the z-score associated to the reliable change index is less than or equal to the threshold. These results are averaged across 1,000 simulated datasets of sizes 10, 20, 30, 40, 50, 100, $150,200,250,500,750$ and 1,000 drawn at random from a simulated population comprising of one million individuals. 
Figure 1

Significance of F-test

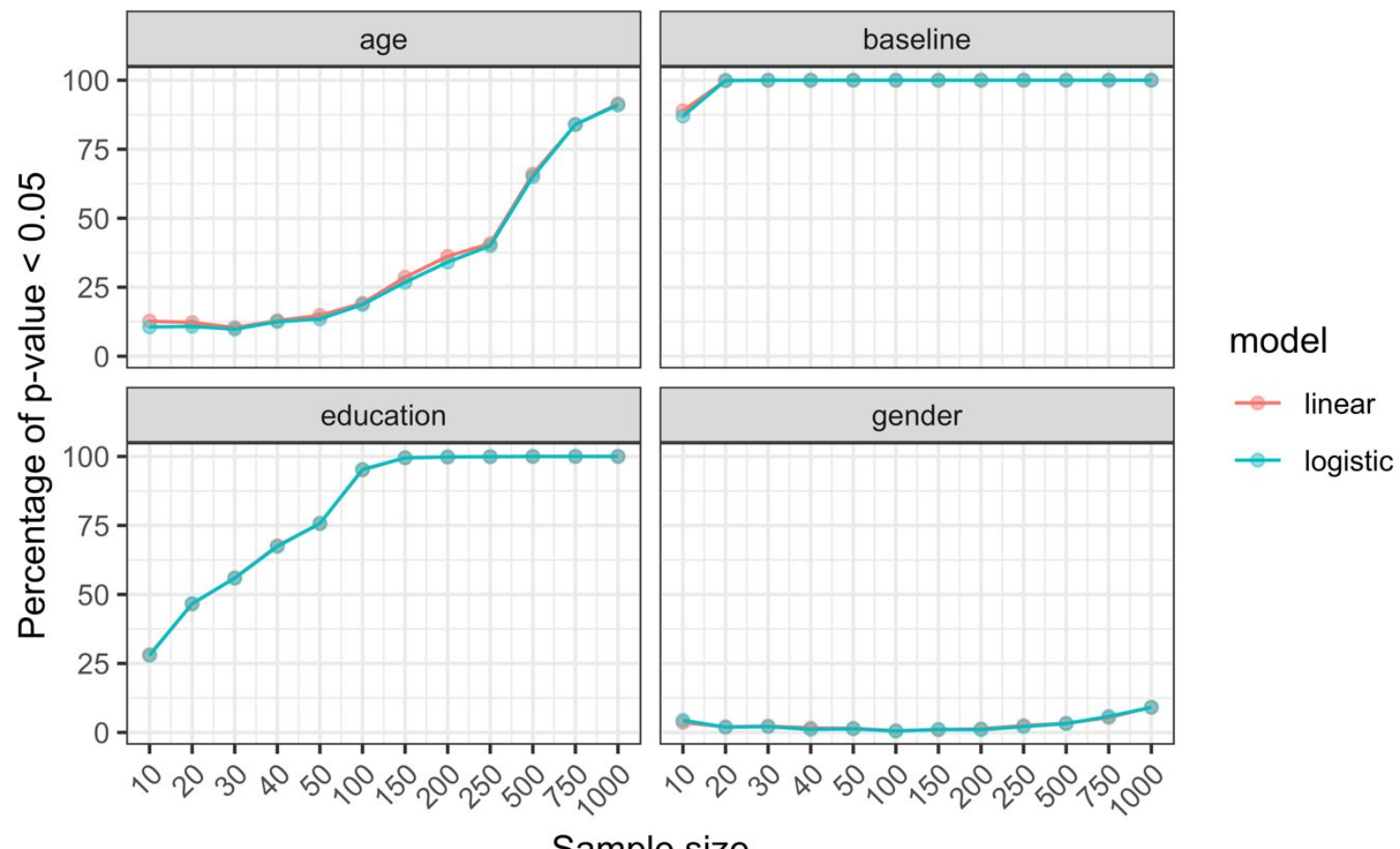

Sample size 
Figure 2

\section{True Positive Rate}
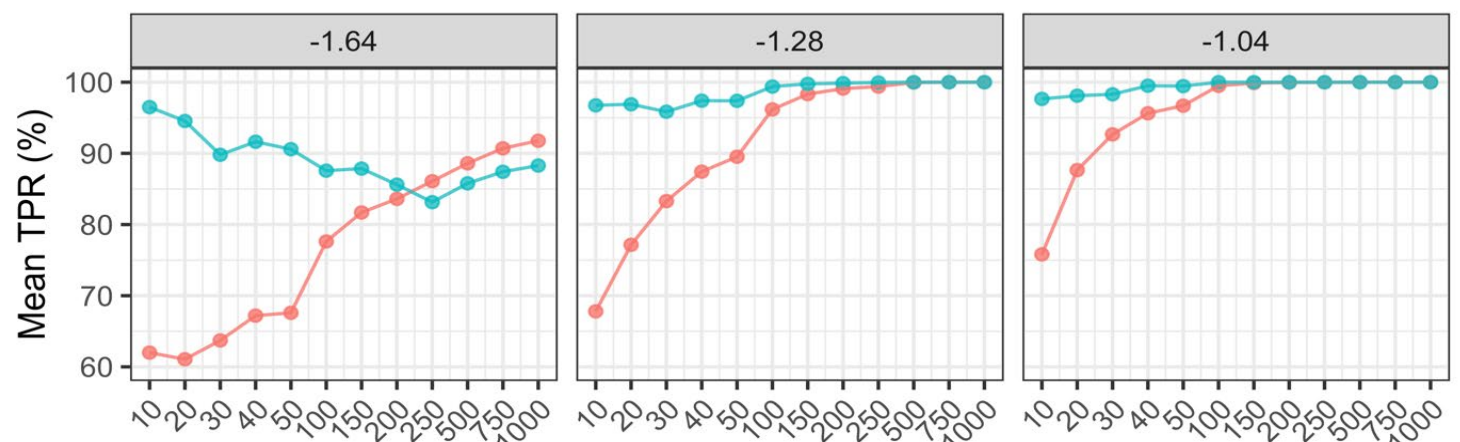

Sample size

True Negative Rate
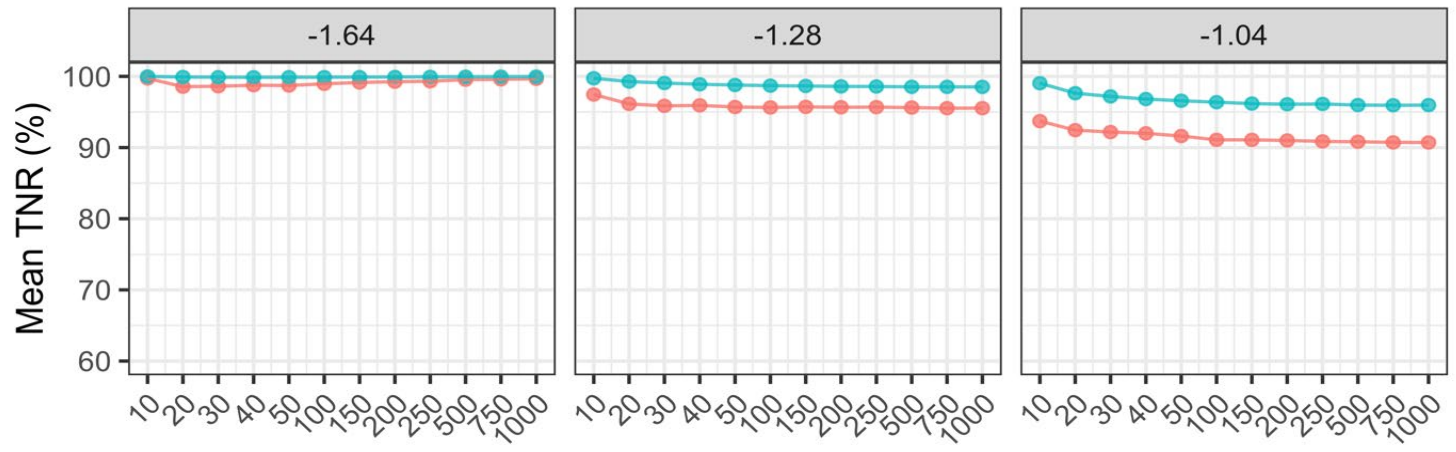

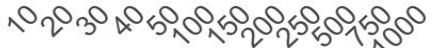

$12^{2} \mathrm{O}^{\circ} \times 2$.

Sample size
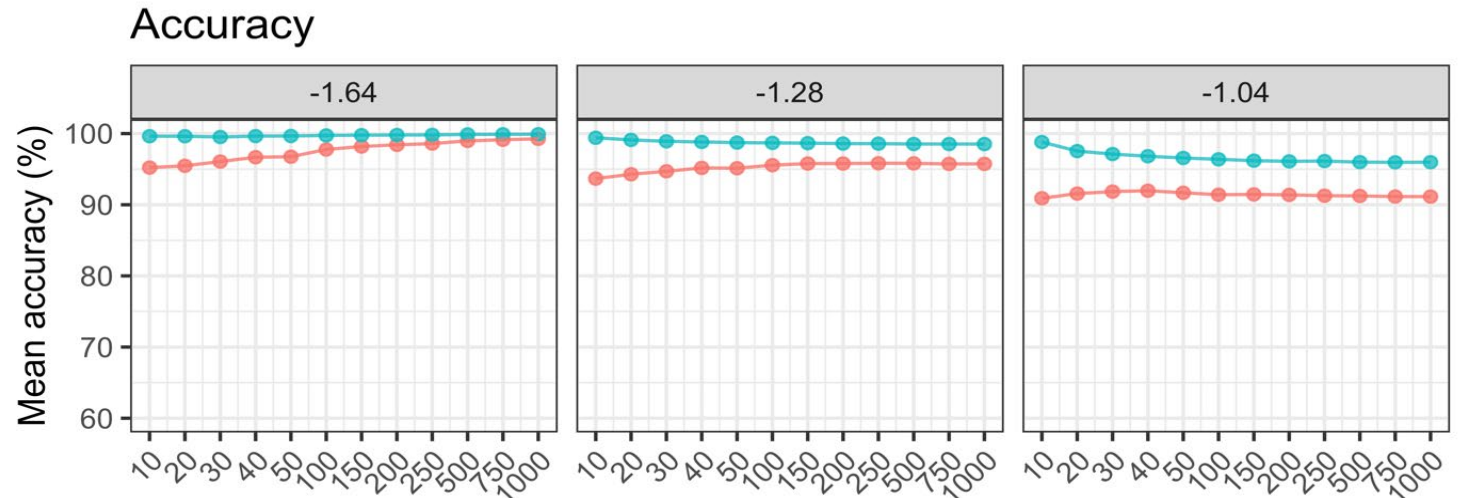

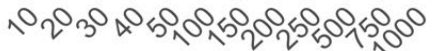

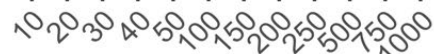

Sample size

model $\multimap-$ linear $\multimap$ logistic 
Supplementary Figure 1: Percentage of points that fell outside of the simulated envelope in the half-normal plot for the deviance residuals (left-hand plot) for the linear and logistic regression models and (right-hand plot) the linear and logistic reliable change index, averaged over 1,000 datasets of sizes 10,20,30,40,50,100,150, $200,250,500,750$ and 1,000 drawn at random from a simulated population comprising of one million individuals.

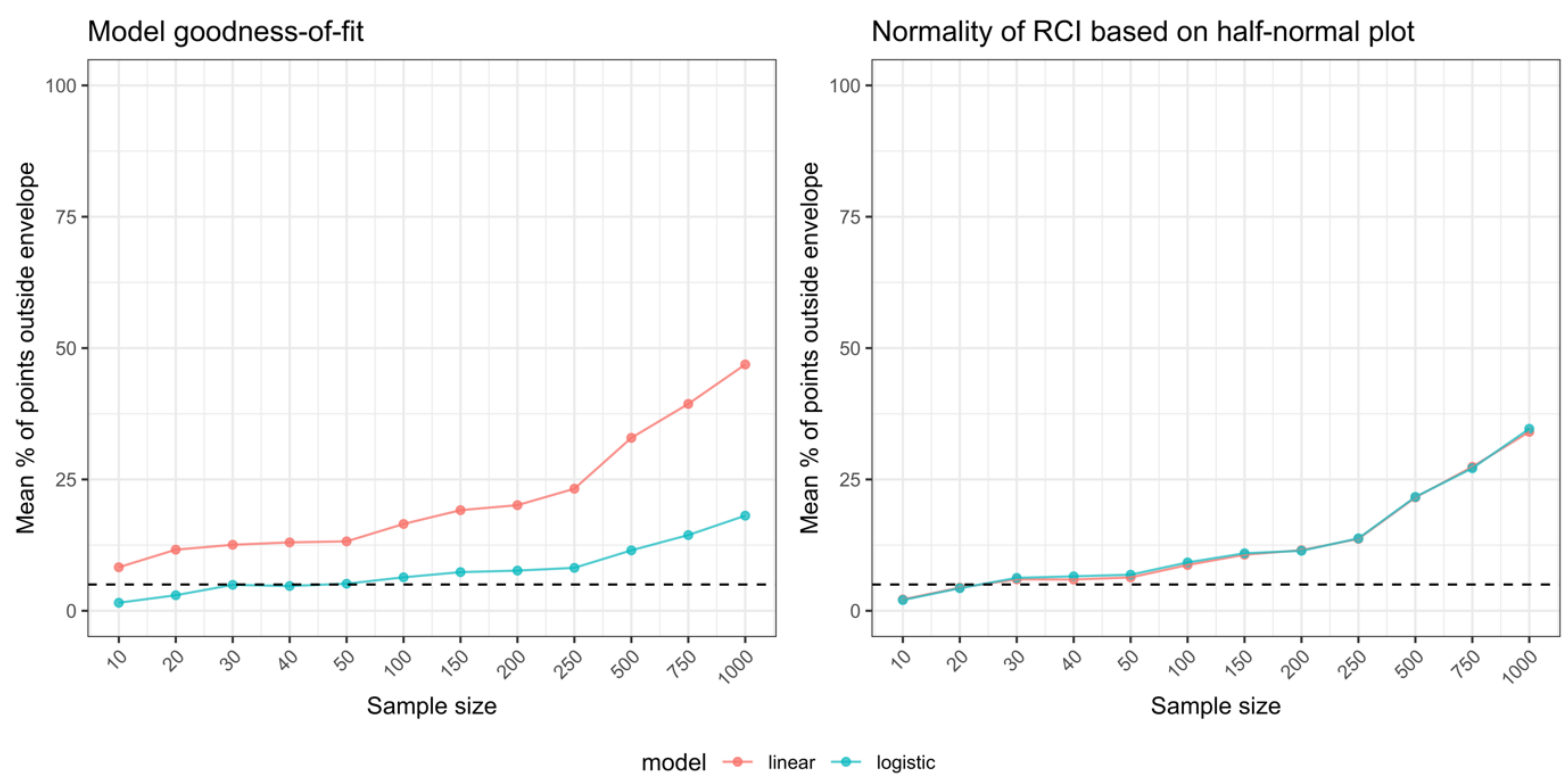


Supplementary Figure 2: Coverage rate of the $90 \%, 95 \%$ and $99 \%$ confidence intervals for the estimated parameters (intercept, baseline score, education, age, and gender) averaged over 1,000 datasets of sizes $10,20,30,40,50,100,150,200,250,500,750$ and 1,000 drawn at random from a simulated population comprising of one million individuals.
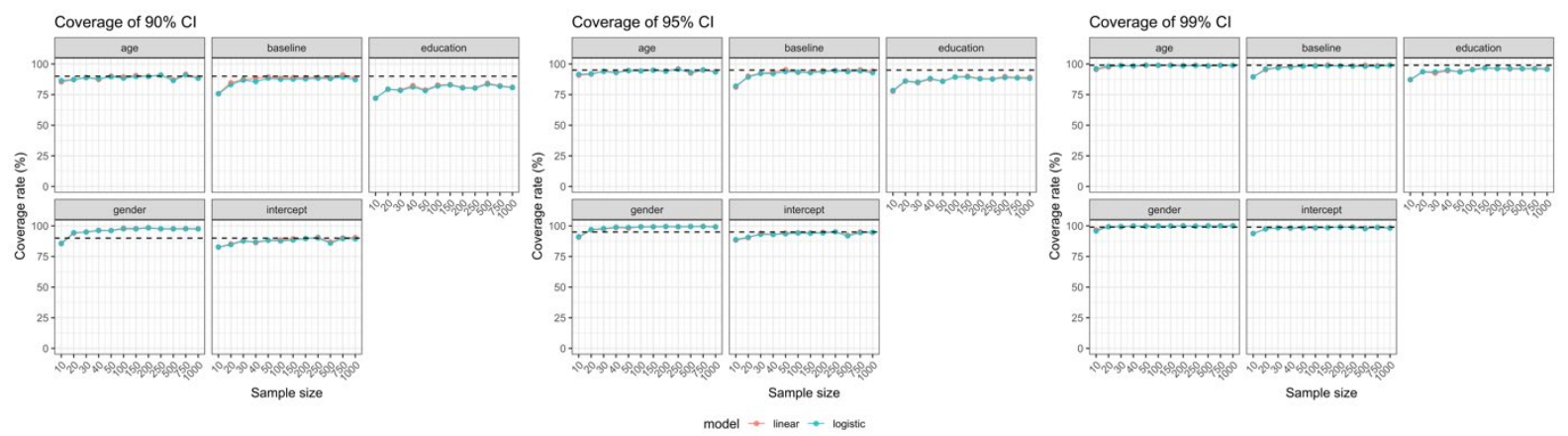
Table 1: Parameter values estimated from the ADNI

dataset from a linear regression model and a logistic

regression model

Linear regression Logistic regression

Parameter estimate estimate

\begin{tabular}{lcc}
\hline Intercept & 2.3102 & -1.5782 \\
Baseline score & 0.6726 & 0.2010 \\
Age & -0.0285 & -0.0087 \\
Education level & 0.0686 & 0.0224 \\
Gender - female & 0.2837 & 0.0894 \\
\hline
\end{tabular}

